\title{
Clinical trials of penicillamine in Indian childhood cirrhosis
}

\author{
M S TANNER, S A BHAVE, A M PRADHAN, AND A N PANDIT \\ Department of Child Health, University of Leicester, and Departments of Pediatrics and Pathology, \\ King Edward Memorial Hospital, Pune, India
}

SUMMARY The outcome in 15 children with advanced Indian childhood cirrhosis (ICC) treated with penicillamine $20 \mathrm{mg} / \mathrm{kg} /$ day was not significantly different from that in untreated children. Among children admitted to a further double blind trial who had ICC but who had not yet developed jaundice or ascites 10 treated with penicillamine and 10 treated with penicillamine plus prednisolone had a significantly improved survival. Fourteen of 29 treated cases made a clinical recovery and were alive 489 to 1460 days from the start of treatment. Biopsy specimens in survivors showed a return to normal liver histology in three, residual fibrosis in six, and inactive micronodular cirrhosis in five. Thus penicillamine, while not shown to be beneficial in advanced ICC, lowered mortality from $93 \%$ to $52 \%$ in preicteric cases of ICC.

The prognosis of Indian childhood cirrhosis (ICC) is poor. ${ }^{1}$ Of cases presenting to the King Edward Memorial Hospital in Pune in 1979 and 1980, 45\% died within four weeks, $74 \%$ within eight weeks, and $86 \%$ within six months. ${ }^{2}$ Suggested treatment has included steroids and gammaglobulins, ${ }^{3}$ levamisole ${ }^{4}$ and several traditional and 'ayurvedic' medicines. The copper chelating agent penicillamine is an obvious option for treatment because of the gross hepatic copper accumulation that is characteristic of ICC. ${ }^{5}$ The poor results of chelation treatment in patients with childhood Wilson's disease who presented with advanced disease ${ }^{7}$ however, suggested that penicillamine could prove disappointing in ICC as most cases present at a very advanced stage. A proper trial of penicillamine was therefore indicated to forestall the introduction of an expensive, potentially toxic, and possibly ineffective treatment.

Two trials were conducted: the first included a number of advanced cases, and results were poor. This led to a second trial to which only patients who had not progressed to the development of jaundice or ascites were admitted. In this second trial a third treatment group received prednisolone in addition to penicillamine; the rationale for the steroids was their reported efficacy by other workers, ${ }^{7}$ the inflammatory component of the hepatic pathology, and the possibility that lysosomal membrane stabilisation could limit copper cytotoxicity.

\section{Subjects and methods}

Children admitted to the King Edward Memorial Hospital in Pune in whom ICC was clinically diagnosed underwent percutaneous liver biopsy if coagulation studies permitted. If the prothrombin time was more than five seconds prolonged parenteral vitamin $\mathrm{K}$ and fresh donor blood were given. The Tru-Cut needle (Travenol) was used with local anaesthaesia and sedation with intravenous diazepam.

ICC was diagnosed if histological examination showed that the following features were present ${ }^{9}$ : hepatocyte necrosis, ballooning, and hyaline inclusions; pericellular intralobular fibrosis; and granular orcein staining of hepatocytes.

FIRST TRIAL OF PENICILLAMINE

Thirty children with histologically confirmed ICC were studied. The nature and prognosis of the disease were explained to parents and other senior family members before requesting admission to the trial. During the trial other medications such as ayurvedic drugs and liver extracts were stopped. Supportive care comprised nutritional advice and supplementation, treatment of bacterial infections, treatment with vitamin $\mathrm{K}$, avoidance of non-trial medications, and conservative management of liver failure.

Fifteen packs were prepared containing 600 tab- 
lets of $50 \mathrm{mg}$ penicillamine, and 15 packs containing identical placebo tablets. The packs were randomly numbered and were assigned to patients in order of admission to the trial. Children received one tablet of penicillamine or placebo per $2.5 \mathrm{~kg} /$ body weight/ day in two doses (equivalent to $20 \mathrm{mg}$ penicillamine/ $\mathrm{kg}$ /day). Urinary protein concentration and blood count were monitored weekly for six weeks and thereafter every six weeks where possible. Compliance with treatment was encouraged by repeated home visits by paramedical health workers. In the surviving children a repeat liver biopsy was performed after six weeks if clinically possible. In those children who died in hospital an immediate postmortem liver biopsy was requested. Full autopsy examinations were not permitted by parents.

\section{SECOND TRIAL}

Altogether 30 children with ICC were admitted to the trial. The criteria for admission were a histologically confirmed diagnosis of ICC and absence of ascites or jaundice. There were three treatment groups who were given penicillamine, or penicillamine plus prednisolone, or a placebo. The dose of penicillamine was $20 \mathrm{mg} / \mathrm{kg} /$ day and of prednisolone $2 \mathrm{mg} / \mathrm{kg} /$ day for four weeks and thereafter 5 $\mathrm{mg} /$ day. Packs containing the drug(s) or placebos were randomly numbered and allocated to patients in order of presentation. In children surviving for more than three months the treatment code was broken and treatment continued with the medication the child was found to be taking.

In the interval between designing the trial and the trial packs becoming available in Pune children who would have met the admission criteria for the trial were treated with penicillamine, penicillamine and prednisolone, or vitamins (placebo) in a manner that was not blind but strictly in rotation.

In survivors of the trial penicillamine was continued until the histology of the liver was normal and hepatic copper concentrations had returned to normal.

\section{DATA HANDLING}

Clinical and standard laboratory variables were recorded on a standard form. A semi quantitative assessment of the features of a liver biopsy specimen was made by two pathologists. Data were stored in Pune by a BBC B microcomputer with purpose written software. ${ }^{10}$ Discs were mailed to Leicester and data transferred to the Leicester University DEC VAX computer. Comparison of baseline characteristics was performed by standard statistical methods. Where the assumptions of normality and constant variance were insecure, non-parametric tests (Mann-Whitney U, Kruskal-Wallis) were used.
Survival analysis was performed by program P1L of the BMDP statistical software package. ${ }^{11}$

\section{LABORATORY METHODS}

The concentration of copper in the liver was assayed in sections of biopsy specimens that had been freeze dried in Pune and transferred to Leicester. A PyeUnicam SP1 atomic absorption spectrophotometer with electrothermal atomisation was used. Standard biochemical and haematological assays were performed in Pune.

\section{Results}

Penicillamine was well tolerated by children with ICC. Although mild proteinuria was common at presentation, this did not increase with treatment and in no child did a rash or marrow suppression necessitate stopping treatment. No complications of the liver biopsy occurred.

\section{FIRST TRIAL}

The groups were well matched in clinical features (table 1). In both groups boys predominated and seven of 15 were jaundiced. Patients treated with penicillamine were slightly younger and two had a palpable gall bladder, which is a poor prognostic feature. ${ }^{12}$ Results of laboratory tests showed no significant differences. Histological examination of the liver showed that no differences existed with respect to portal tract features (fibrosis, oedema, cellularity, bile duct reduplication, bile plugs, disruption of the limiting plate) or parenchymal features (fibrosis, necrotosis, hyaline inclusions, cholestasis, fat infiltration, inflammatory infiltrate, orcein staining).

Of the 30 children, only nine survived three months, and only four one year. While the median survival in treated children (58 days) was longer than in untreated (26 days) this difference was not significant. One child in each group survived to the second year then died suddenly at home: one child after measles and one after severe haematemesis. One child in each group has survived at the time of writing (more than six years). One of these, treated with penicillamine, was unusual in that his biopsy specimen at presentation showed fat infiltration in addition to the features of ICC. Follow up liver biopsy specimens showed his hepatic copper falling from $2444 \mu \mathrm{g}$ copper/g dry weight tissue at presentation to 1935 at two months, 1569 at four months, 884 at eight months, and 36 at 20 months.

\section{SECOND TRIAL}

No significant differences existed between treatment groups for clinical, laboratory, or histological 
Table 1 Clinical and laboratory data at presentation in first trial. Figures are numbers or mean (SEM)

\begin{tabular}{|c|c|c|}
\hline & $\begin{array}{l}\text { Group given } \\
\text { penicillamine } \\
\text { (13 boys, } \\
\text { two girls) }\end{array}$ & $\begin{array}{l}\text { Group given } \\
\text { placebo } \\
\text { (11 boys, } \\
\text { four girls) }\end{array}$ \\
\hline \multicolumn{3}{|l|}{ No of children who had: } \\
\hline Previously used steroids & 3 & 4 \\
\hline Jaundice & 7 & 7 \\
\hline Dilated abdominal wall veins & 6 & 5 \\
\hline Palpable gall bladder & 2 & 0 \\
\hline Age (months) & $17 \cdot 1(2 \cdot 2)$ & $20 \cdot 9(2 \cdot 2)$ \\
\hline Weight $(\mathrm{kg})$ & $7 \cdot 5(0 \cdot 4)$ & $8.5(0.4)$ \\
\hline Height $(\mathrm{cm})$ & $72 \cdot 5(2 \cdot 2)$ & $75 \cdot 8(1.8)$ \\
\hline Duration of abdominal distension (weeks) & $7 \cdot 1(2 \cdot 2)$ & $12 \cdot 9(3 \cdot 4)$ \\
\hline Liver size $(\mathrm{cm})$ & $7 \cdot 5(0 \cdot 6)$ & $7 \cdot 0(0 \cdot 5)$ \\
\hline Spleen size $(\mathrm{cm})$ & $3.9(0 \cdot 6)$ & $4 \cdot 0(0 \cdot 6)$ \\
\hline Total bilirubin ( $\mathrm{mg} / \mathrm{l})$ & $25 \cdot 7(4 \cdot 4)$ & $25 \cdot 6(4 \cdot 3)$ \\
\hline Conjugated bilirubin (mg/l) & $10 \cdot 9(3 \cdot 0)$ & $9 \cdot 6(2 \cdot 1)$ \\
\hline Serum glutamic pyruvic transaminase (IU/I) & $117 \quad(19)$ & $115 \quad(16)$ \\
\hline Prothrombin time (seconds prolonged) & (1) & $(3)$ \\
\hline Albumin $(g / l)$ & (3) & (1) \\
\hline Alkaline phosphatase (IU/l) & $100 \quad(17)$ & $92 \quad(14)$ \\
\hline Haemoglobin $(g / l)$ & $89 \cdot 0(5 \cdot 0)$ & $88.0(5 \cdot 0)$ \\
\hline White cell count $\times 10^{9} / 1$ & $14 \cdot 8(1.6)$ & $12.4(0.8)$ \\
\hline Platelets $\times 10^{9} / 1$ & 203 & 251 \\
\hline Liver copper ( $\mu \mathrm{g} / \mathrm{g}$ dry weight) & $1802 \quad(152)$ & $(219)$ \\
\hline
\end{tabular}

Table 2 Clinical and laboratory data at presentation in second trial. Figures are numbers or mean (SEM)

\begin{tabular}{|c|c|c|c|}
\hline & $\begin{array}{l}\text { Group given } \\
\text { penicillamine } \\
\text { (seven boys, } \\
\text { three girls) }\end{array}$ & $\begin{array}{l}\text { Group given } \\
\text { penicillamine } \\
\text { plus prednisolone } \\
\text { (nine boys, } \\
\text { one girl) }\end{array}$ & $\begin{array}{l}\text { Group given } \\
\text { placebo } \\
\text { (eight boys, } \\
\text { two girls) }\end{array}$ \\
\hline \multicolumn{4}{|l|}{ No of children who had: } \\
\hline Steroids & 0 & 1 & 2 \\
\hline Jaundice & 0 & $3^{*}$ & 0 \\
\hline Ascites* & 3 & 1 & 3 \\
\hline Dilated abdominal wall veins & 2 & 1 & 2 \\
\hline Palpable gall bladder & 0 & 0 & 0 \\
\hline Age (months) & $18 \cdot 7 \quad(2 \cdot 2)$ & $15 \cdot 4(2 \cdot 4)$ & $21 \cdot 1(2 \cdot 2)$ \\
\hline Weight (kg) & $7 \cdot 8 \quad(0 \cdot 7)$ & $8 \cdot 1(0 \cdot 6)$ & $9 \cdot 2(0 \cdot 6)$ \\
\hline Height $(\mathrm{cm})$ & (1) & $73 \quad(3)$ & $77 \quad(1)$ \\
\hline Duration of abdominal distension (weeks) & $4.5 \quad(1.0)$ & $6 \cdot 9(2 \cdot 5)$ & $5 \cdot 2(1 \cdot 3)$ \\
\hline Liver size $(\mathrm{cm})$ & $56 \cdot 5 \quad(5 \cdot 6)$ & $62 \cdot 5(5 \cdot 8)$ & $66 \cdot 5(7 \cdot 6)$ \\
\hline Spleen size $(\mathrm{cm})$ & $29.5 \quad(7.4)$ & $33 \cdot 5(7 \cdot 2)$ & $29 \cdot 5(5 \cdot 3)$ \\
\hline Abdominal girth $(\mathrm{cm})$ & $44 \cdot 3 \quad(2 \cdot 0)$ & $41 \cdot 9(17)$ & $44 \cdot 1(12)$ \\
\hline Total bilirubin $(\mathrm{mg} / \mathrm{l})$ & $11.4 \quad(1.8)$ & $14 \cdot 5(4 \cdot 7)$ & $20 \cdot 4(3.9)$ \\
\hline Conjugated bilirubin $(\mathrm{mg} / \mathrm{l})$ & $7.4 \quad(0.7)$ & $8 \cdot 3(3.5)$ & $8.4(0.9)$ \\
\hline Serum glutamic pyruvic transaminase (IU/l) & $91 \quad(9)$ & $77 \quad(15)$ & $110 \quad(25)$ \\
\hline Prothrombin time (seconds prolonged) & $4 \cdot 5 \quad(1 \cdot 0)$ & $4 \cdot 4(1 \cdot 6)$ & $5 \cdot 5(0 \cdot 8)$ \\
\hline Albumin $(g / 1)$ & $(2 \cdot 1)$ & $35 \quad(5 \cdot 8)$ & $34 \quad(2 \cdot 5)$ \\
\hline Haemoglobin $(\mathrm{g} / \mathrm{l})$ & $100 \cdot 0 \quad(8.0)$ & $101 \cdot 0(9 \cdot 0)$ & $97 \cdot 0(6 \cdot 0)$ \\
\hline White cell count $\times 10^{9} / 1$ & $11 \cdot 8 \quad(2 \cdot 1)$ & $11 \cdot 3(1 \cdot 3)$ & $11 \cdot 5(2 \cdot 9)$ \\
\hline Platelets $\times 10^{-9} / 1$ & $(28)$ & 207 & $168 \quad(16)$ \\
\hline Liver copper $(\mu \mathrm{g} / \mathrm{g}$ dry $w \mathrm{t})$ & (263) & 1707 & 1044 \\
\hline
\end{tabular}

${ }^{*}$ Jaundice or ascites were absent at presentation but became apparent before treatment was started. 
variables (table 2). Despite the earlier stage of their disease compared with children in the first trial, nine of 10 untreated children died within 181 days, median 58 days. One untreated child recovered spontaneously and was alive at the time of writing ( $2 \cdot 7$ years).

At the time of writing five children treated with penicillamine and five treated with penicillamine plus prednisolone survive 489-1460 days from the start of treatment. Life table analysis showed a significantly improved survival in both treatment groups compared with the group taking the placebo $(p<0.05)$. No difference in survival existed between the two treatment groups. When these two groups were pooled the difference in survival between treated children $(n=20)$ and the group taking placebo $(n=10)$ was highly significant $(\mathrm{p}<0.005)$ (table 3).

Thirteen children who were eligible for admission to the second trial but who presented before the treatment packs were available were allocated to treatment groups in rotation. Inclusion of these children (five treated with penicillamine, four

Table 3 Comparison of survival distribution in treated and untreated ICC

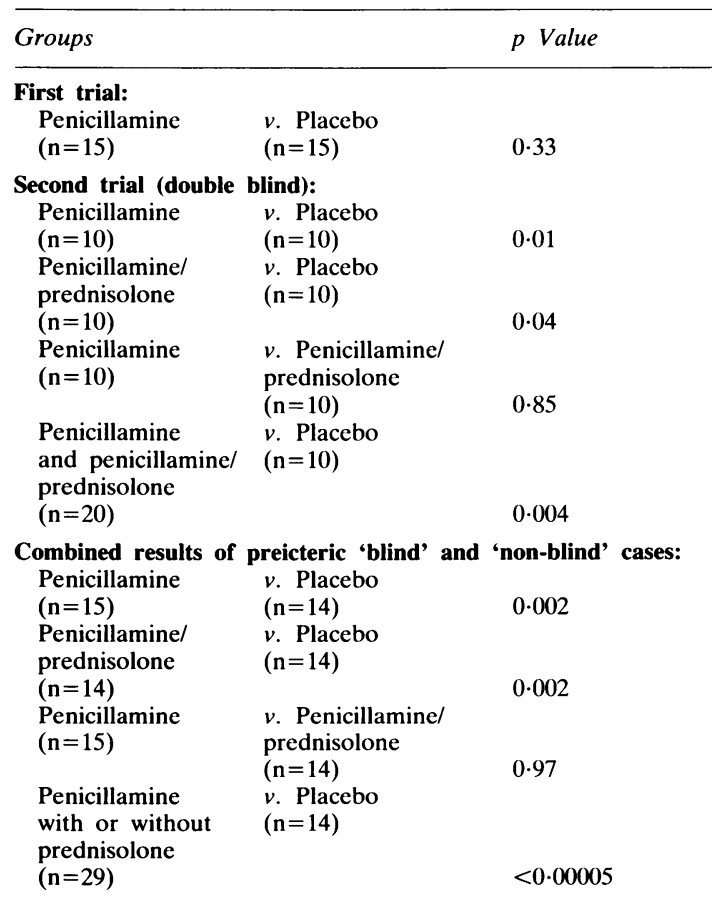

Life table analysis was performed using program P1L of the BMDP package, ${ }^{11}$ and the equality of survival between groups tested by the Mantel-Cox statistic treated with penicillamine and prednisolone, and four given only supportive treatment and vitamins) did not introduce any differences between groups at entry but further increased the significance of the difference between survival in treated and untreated children (table 3 ).

Taking all the 29 children treated with penicillamine or penicillamine and prednisolone, a comparison was made between the 14 who survived and the 15 who died to determine whether there were features at entry to the trial that could have predicted a better outcome. Five of 15 non-survivors developed jaundice between presentation and the start of treatment. Non-survivors had larger spleens, higher serum bilirubin concentrations, longer prothrombin times, and higher hepatic copper concentrations, although these differences did not achieve significance (table 4).

There were 33 liver biopsies performed at follow up in the 14 surviving children. Because of the distance of their homes from Pune and the reluctance of their parents to allow further stays in hospital after clinical improvement biopsies were performed irregularly but all children had one follow up examination and six had three. While five of 14 biopsy specimens taken within six months of starting treatment still showed features of ICC a transition was seen in successive biopsy specimens through the following stages: (i) cirrhosis with thick septae and continued inflammatory infiltration; (ii) inactive micronodular cirrhosis with thin septae, no inflammation, and healthy parenchyma; (iii) incomplete fibrous septae partially dividing a healthy parenchyma; and (iv) almost normal appearances with mild portal fibrosis. At the latest follow up three children had almost normal biopsy specimens, six had incomplete fibrous septae, and five had inactive micronodular cirrhosis.

The concentration of copper in the liver fell at varying rates in different children and was $<100 \mu \mathrm{g} / \mathrm{g}$ dry weight in only five of the 12 biopsy specimens taken seven to 36 months after starting treatment (table 5).

\section{Discussion}

Before conducting the first trial careful consideration was given to the need for a placebo group. Because of continuing controversy as to the definition and prognosis of ICC $^{1}$ published data would not form an accurate comparison with the treated group. Cases of ICC previously treated in Pune were also not acceptable because the trial protocol included a standard of supportive care that was provided without charge to the family, and which had not previously been available. ${ }^{2}$ For the second trial, 
Table 4 Comparison of clinical and laboratory features between survivors and non-survivors in preicteric children with ICC treated with penicillamine and penicillaminelprednisolone. Figures are numbers or mean (SEM)

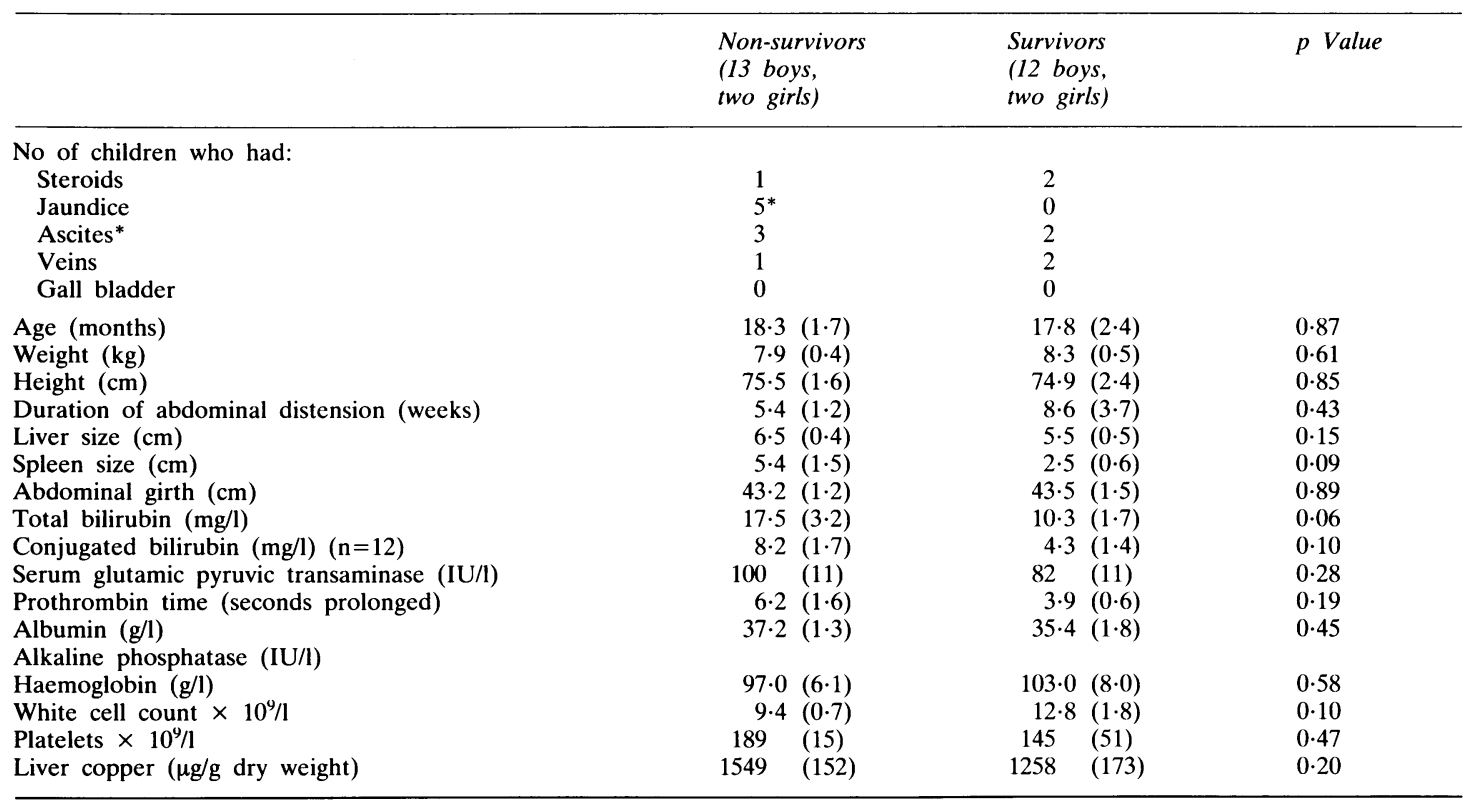

*Jaundice or ascites were absent at presentation but became apparent before treatment was started.

Table 5 Histological findings and concentration of copper in the liver in follow up biopsy specimens of children with ICC treated with penicillamine and penicillamine/prednisolone

\begin{tabular}{|c|c|c|c|c|c|}
\hline & \multicolumn{5}{|c|}{ Months from presentation } \\
\hline & $0-6$ & $7-12$ & $13-24$ & $25-36$ & $>36$ \\
\hline Indian childhood cirrhosis & 5 & & & & \\
\hline Cirrhosis with thick septae and inflammation & 3 & 1 & & & \\
\hline Micronodular cirrhosis with thin septae & 5 & 2 & 3 & 3 & 1 \\
\hline Incomplete fibrous septae with normal parenchyma & 1 & 2 & 1 & & 2 \\
\hline Mild portal fibrosis & & & 1 & 2 & 1 \\
\hline \multicolumn{6}{|l|}{ Liver copper $\mu \mathrm{g} / \mathrm{g}$ dry weight } \\
\hline No & 11 & 4 & 4 & 4 & 2 \\
\hline Mean & 977 & 385 & 82 & 143 & 365 \\
\hline Range & 68-1973 & $28-895$ & $10-161$ & $33-323$ & 325,40 \\
\hline
\end{tabular}

in which the cases were by definition at a less advanced stage of the disease, data as to prognosis without treatment did not exist, and so a placebo group was again necessary. As a difference between treated and untreated cases appeared, termination of the trial was considered. Interim results were presented for discussion at two scientific meetings and continuation of the trial to achieve significance was urged by those taking part. ${ }^{13}$ The significantly improved survival in patients treated with penicillamine seen in the second trial now makes it unacceptable for any future trial of penicillamine to include a placebo group.

The untreated children in this study show the grim prognosis and fulminant course of ICC. Even those children who did not have ascites or jaundice at presentation declined rapidly, 13 of 14 dying within 165 days. Before the first trial the mortality of ICC 
without treatment was estimated to be about $90 \%$, and a power calculation shows that the chance of detecting a true halving of this mortality would be $90 \%$ at the $5 \%$ significance level if 15 children were included in both groups. The fact that there was no difference in survival between the two groups in the first trial has two possible explanations. Firstly, penicillamine may be genuinely ineffective in advanced ICC. The extensive hepatocellular damage seen in this condition, the clinical condition of the affected children, and the failure of penicillamine to improve survival in Wilson's disease presenting with fulminant hepatic failure all support that conclusion. Secondly, penicillamine may have a therapeutic effect that would only become apparent in a larger trial. Because of the disappointing results in the first trial, subsequent cases presenting with advanced ICC have not been treated with penicillamine. With the results of the second trial now available treatment in advanced ICC must be reconsidered.

In the second trial penicillamine alone and in combination with prednisolone improved survival. As there were no differences between results in the two treatment groups it is legitimate to make a comparison between all treated children, where 14 of 29 survived, with the sole survivor of 14 untreated children. Two further treated children made a clinical recovery but died in the second year (at 440 and 448 days of treatment) from the effects of portal hypertension and cirrhosis. Treated children had few side effects of penicillamine. Compliance, as judged by the number of tablets remaining when the family members were visited, was good.

Although absence of jaundice or ascites were criteria for admission to the second trial, jaundice did become apparent in six children in the interval between admission to hospital and the start of treatment. Five of these were in active treatment groups (one taking penicillamine, four penicillamine plus prednisolone), and none survived (table 4). This further emphasises the rapid course of ICC and the need to start chelation treatment promptly.

The rationale for penicillamine use was removal of copper from the liver by chelation, and treated patients indeed showed a progressive fall in hepatic copper concentration. This carries the implication that more effective decoppering agents such as ammonium thiomolybdate may have a role in advanced disease. ${ }^{14}{ }^{15}$ Other modes of action are, however, possible. Firstly, penicillamine as a sulphydryl donor may regenerate reduced glutathione so protecting against cytotoxic injury. If this is so then other agents with similar actions such as acetyl cysteine may have an acute therapeutic role. Secondly, penicillamine may interfere with collagen synthesis by preventing cross linking of collagen chains. ICC is characterised by aggressive pericellular intralobular fibrosis and serum markers of collagen biosynthesis are present in high concentrations. ${ }^{16}$ Although it seems unlikely that an effect on collagen could achieve so rapid a difference in survival between treated and untreated groups, it may contribute to the reduction in fibrosis seen in treated patients in subsequent biopsy specimens. Thirdly, penicillamine may reduce the concentration of circulating immune complexes. Unpublished results (Vergani et al) show increased concentrations of serum immune complexes in ICC, their concentration increasing with disease severity. Their presence in other liver disorders, however, suggests that they are a result rather than a cause of liver cell injury. Fourthly, there is increasing interest by rheumatologists in penicillamine as an antiinflammatory drug, its action mediated by the superoxide dismutase like action of the copperpenicillamine chelate. ${ }^{1718}$ The lack of side effects of penicillamine in ICC is similar to Wilson's disease but contrasts with rheumatoid arthritis. ${ }^{18}$

In the presence of penicillamine, prednisolone has not been shown to confer additional benefit. Conversely, it was not associated with adverse effects, and with the completion of the trial it was decided to continue treatment of preicteric cases with penicillamine either with or without prednisolone in alternate allocation to see whether there is a difference from larger patient groups. As the number of children treated with penicillamine increases it will become possible to define more accurately favourable and unfavourable prognostic features (table 4) by discriminant analysis.

The clinical recovery from ICC was associated with progressive improvement in histology. These results (to be presented in more detail elsewhere) showed a progressive evolution from ICC to: (i) active cirrhosis with thick inflammatory septae; (ii) inactive micronodular cirrhosis; and (iii) an apparently normal parenchyma with thin fibrous septae. There are few other diseases in which established cirrhosis has been shown to be reversible. The evolution of ICC to inactive micronodular cirrhosis raises the possibility that some children with cryptogenic cirrhosis who are older than those with ICC at presentation may have had a similar aetiology. The occurrence of ICC and inactive micronodular cirrhosis in siblings and a history of copper ingestion in infancy in some cases of inactive micronodular cirrhosis support this conclusion. ${ }^{19}$

While ICC may be totally preventable by change in feeding practices in infancy, ${ }^{2021}$ the present results indicate that treatment of preicteric patients with penicillamine improves survival from $10 \%$ to 
$45 \%$ and provides a basis for further improvements in treatment.

We thank the Wellcome Trust for generous financial support; $\mathrm{Dr}$ BA Gennery of Lilly Industries and Ms DA Garrood of the Boots Company for preparing and randomising the trial packs; Dr Kent Woods for invaluable statistical advice; colleagues in Pune for referring cases; and Mrs Anne Gilbert for typing the manuscript.

\section{References}

1 Tanner MS. Indian childhood cirrhosis. In: Meadow SR, ed. Recent advances in peadiatrics 8. London: Churchill Livingstone, 1986:103-20.

2 Bhave SA, Pandit AN, Pradhan AM, et al. Liver disease in India. Arch Dis Child 1982;57:922-8.

${ }^{3}$ Raju VB, Sundaravalli N, Sriramachari S. Indian childhood cirrhosis: clinical features, prognosis, and treatment. Indian J Pediatr 1980;47:537-41.

${ }^{4}$ Kalra V, Ghai OP, Roy S. Immuno-modulation: a therapeutic approach to Indian childhood cirrhosis. Indian Pediatr 1981;18:247-52.

5 Tanner MS, Portmann B, Mowat AP, et al. Increased hepatic copper in Indian childhood cirrhosis. Lancet 1979;i:1203-5.

${ }^{6}$ Popper H, Goldfisher S, Sternlieb I, Nayak NC, Madhavan TV. Cytoplasmic copper and its toxic effects- studies in Indian childhood cirrhosis. Lancet 1979;i:1205-8.

${ }^{7}$ Nazer H, Ede RJ, Mowat AP, Williams R. Wison's disease: clinical presentation and use of prognostic index. Gut 1986;27:1377-81.

${ }^{8}$ Nayak NC, Ramalingaswami V. Indian childhood cirrhosis. Clin Gastroenterol 1975;4:333-49.

9 Tanner MS, Portmann B. Indian childhood cirrhosis. Arch Dis Child 1981;56:4-6.

${ }^{10}$ Williams THC, Sargent GF, Ashmore S, John I, Tanner MS. Microcomputer data acquisition in a developing country with transfer to a mainframe computer for analysis. In: Vicary FR, ed. Computers in gastroenterology. Berlin: Springer-Verlag (in press).
1 BMDP statistical software. Berkeley, University of California Press, 1983:557-69.

12 Pandit AN, Bhave SA, Sidhaye DG, Datar GV. Palpable gall bladder in Indian childhood cirrhosis. Indian Pediatr 1981;18:905-8.

13 Tanner MS, Sidhaye DG, Bhave SA, Pradhan AM. Interim results show major improvement in survival in pre-icteric Indian childhood cirrhosis (ICC) treated with d-penicillamine. Gut 1984;25:A1134.

${ }^{14}$ Gooneratne SR, Howell JMcC, Gawthorne JM. Intravenous administration of thiomolybdate for the prevention and treatment of chronic copper poisoning in sheep. $\mathrm{Br} J$ Nutr 1981;46:457-67.

15 Gooneratne SR, Howell JMcC, Gawthorne JM. An investigation of the effects of intravenous administration of thiomolybdate on copper metabolism in chronic $\mathrm{Cu}$-poisoned sheep. Br J Nutr 1981;46:469-80.

16 Trivedi P, Risteli J, Risteli L, et al. Serum type III procollagen and basement membrane proteins as non-invasive markers of hepatic pathology in Indian childhood cirrhosis. Hepatology (in press).

17 Lengfelder E, Fuch C, Yonner M, Weser U. Functional aspects of the superoxide dismutative action of Cu-penicillamine. Biochim Biophys Acta 1979;567:492-502.

18 Roberts NA. Robinson PA. Copper chelates of antirheumatic and anti-inflammatory agents: their superoxide dismutase-like activity and stability. Br J Rheumatol 1985;24:128-36.

19 Pradhan AN, Talbot IC, Tanner MS. Indian childhood cirrhosis and other cirrhoses of Indian children. Pediatr Res 1983;17:435.

20) Tanner MG, Kantarjian AH, Bhave SA, Pandit AN. Early introduction of copper-contaminated animal milk feeds as a possible cause of Indian childhood cirrhosis. Lancet 1983;ii: 992-5.

21 Bhave SA, Pandit AN, Tanner MS. Comparison of feeding history of children with Indian childhood cirrhosis and paired controls. J Pediatr Gastroenterol Nutr 1987;6:562-7.

Correspondence to Dr MS Tanner, Department of Child Health, University of Leicester, Clinical Sciences Building, PO Box 65, Leicester.

Received 5 August 1987 